\title{
Lysosomes spill the signal
}

Two new studies in Nature

Immunology have uncovered a mechanism of innate immune activation that might underlie conditions such as Alzheimer's disease and silicosis, a progressive lung illness caused by the inhalation of silica crystals.

Many diseases with underlying inflammatory pathology are associated with increased levels of the pro-inflammatory cytokine interleukin-1 $\beta$ (IL-1 $\beta$ ). IL-1 $\beta$ maturation is controlled by caspase-1, the activation of which is tightly regulated by the NALP3 (NACHT-, LRR- and pyrin-domain-containing protein 3) inflammasome. The authors of these two studies show that the phagocytosis of crystals, such as silica and aluminium salts, or of aggregates of the peptide amyloid- $\beta$, which contribute to the pathology of Alzheimer's disease, induced the activation of the NALP3 inflammasome and IL- $1 \beta$ maturation. Uptake of these particles by macrophages caused the swelling, de-stabilization and rupture of lysosomes that contained the phagocytosed matter. Lysosomal rupture is associated with the release of proteases into the cytoplasm and, in both of these studies, release of the protease cathepsin B was identified as an activator of the NALP3 inflammasome.

Latz and colleagues went on to show that lysosomal acidification was crucial for the activation of NALP3, and that lysosomal rupture

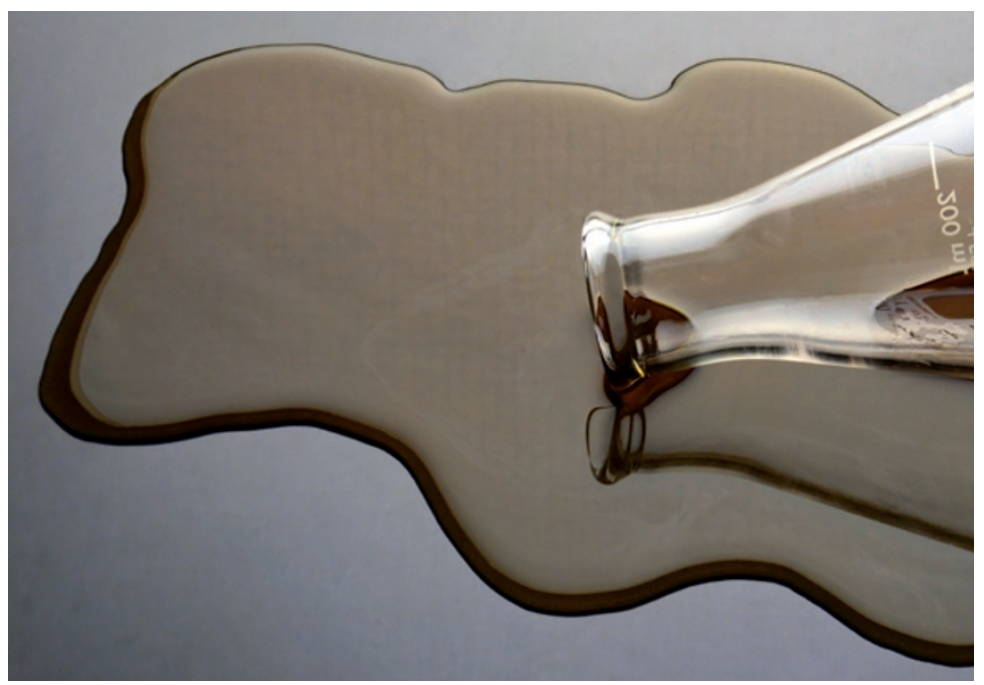

alone - induced by isotonic shock or by an agent known to cause lysosomal damage - was sufficient to activate the NALP3 inflammasome. Therefore, the release of lysosomal contents into the cytoplasm, and in particular of cathepsin B, acts as a danger signal to trigger the activation of the inflammasome and the downstream maturation of IL-1 $\beta$. Golenbock and colleagues further showed that inflammasome activation is responsible for the capacity of microglial cells to mediate pro-inflammatory, chemotactic and neurotoxic effects in response to amyloid- $\beta$.

The evidence provided by Latz and colleagues mechanistically links silica crystals with the inflammatory processes that underlie silicosis, whereas that of Golenbock's group has expanded the understanding of innate immune mechanisms that are triggered by amyloid- $\beta$ in Alzheimer's disease. Together, this work has characterized a new pathway that leads to the activation of the NALP3 inflammasome, linking it to the pathology of these and potentially many other inflammatory diseases.

Sarah Allan

ORIGINAL RESEARCH PAPERS Hornung, V. et al. Silica crystals and aluminum salts activate the NALP3 inflammasome through phagosomal destabilization. Nature Immunol. 11 July 2008 (doi:10.1038/ni.1631) | Halle, A. et al. The NALP3 inflammasome is involved in the innate immune response to amyloid- $\beta$. Nature Immunol. 11 July 2008 (doi:10.1038/ni.1636) 